\title{
Water Temperature Distribution When We Take a Bath in the Bathtub
}

\author{
Yiying Li \\ North China Electric Power University, Baoding, 071000, China \\ zxcva123@163.com
}

Keywords: Temperature, Bathtub, Three-Dimensional, Fourier's Law

\begin{abstract}
Through the analysis of the water temperature distribution affected by the shape, volume, temperature of the tub and the person, this model finds the reason why it is so difficult to keep an evenly expecting temperature during bathing time. Furthermore, according to the water temperature distribution, this paper proposed an optimization model for designing best strategy the person in the bathtub can adopt with the combination of the heat loss considered.
\end{abstract}

\section{Introduction}

Having a warm and comfortable bath is a wonderful experience after a day of tough work. Unfortunately hot water in bathtub can't keep in excellent temperature for long time because of thermal conduction between water surface and the air. So we usually add constant hot water from the faucet to reheat the bathing water which is a usual solution. To find a better way to enjoy bath, water temperature distribution inside model is established in the next section, after a statement of the basic problem.

\section{Water Temperature Distribution Inside}

This article propose the model to investigate the temperature distribution of water when just water in the bathtub.

Heat Conduction. On a microscopic scale, conduction occurs within a body considered as being stationary; this means that the kinetic and potential energies of the bulk motion of the body are separately accounted for. Internal energy diffuses as rapidly moving or vibrating atoms and molecules interact with neighboring particles, transferring some of their microscopic kinetic and potential energies, these quantities being defined relative to the bulk of the body considered as being stationary. Heat is transferred by conduction when adjacent atoms or molecules collide, or several electrons move backwards and forwards from atom to atom in a disorganized way so as not to form a macroscopic electric current, or as phonons collide and scatter. Conduction is the most significant means of heat transfer within a solid or between solid objects in thermal contact. Conduction is greater in solids because the network of relatively close fixed spatial relationships between atoms helps to transfer energy between them by vibration[1].

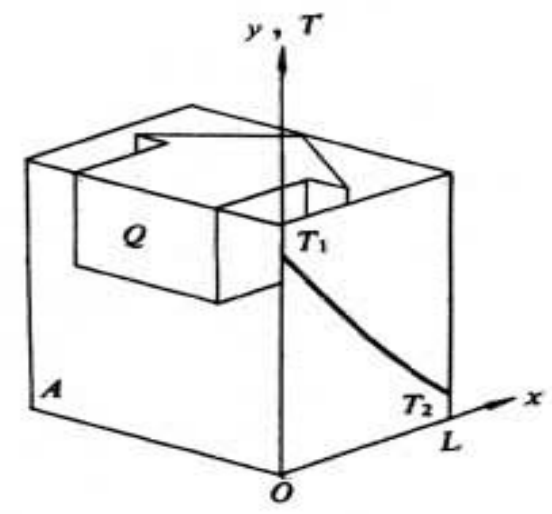

Fig.1 heat flows from high-temperature point to the lower

Fourier's law[2]. The law of heat conduction, also known as Fourier's law, states that the time rate of heat transfer through a material is proportional to the negative gradient in the temperature 
and to the area, at right angles to that gradient, through which the heat flows. We can state this law in two equivalent forms: the integral form, in which we look at the amount of energy flowing into or out of a body as a whole, and the differential form, in which we look at the flow rates or fluxes of energy locally.

Preliminary model. Our preliminary model of bathtub water is based on Fourier' Law. In bathtub water, since the temperature of deferent points of the whole system is not exactly the same, the heat at the higher point of the heat will flow to the point of low temperature, which is called the heat conduction. The heat conduct process is always shown as the temperature changes with time and position. Here we select a curved surface $S$ inside the bathtub, which contents space called $V$. We define $u(x, y, z, t)$ as the temperature of the point $M(x, y, z)$ at the moment $t$, and take $n$ as the normal vector of curved surface $S$.

It's easy to known from Fourier' Law in thermodynamics[3], over a period time of $d t$, the heat $d Q$ flows through an area element $d S$ proportional to $d t, d S$ and $\frac{\partial u}{\partial n}$ as the derivative of water temperature $u$ along normal direction of $d S$, which is shown below:

$$
d Q=-k \frac{\partial u}{\partial n} d S d t=-k(\operatorname{grad} u)_{n} d S d t=-k \operatorname{grad} \cdot u \cdot d S d t(1)
$$

In which $k=k(x, y, z)$ is thermal conductivity. And $k$ is constant for water is isotropic and homogeneous.

According to the relation above, from moment $t_{1}$ to moment $t_{2}$, the total heat flows through curve $S$ into space $V$ is:

$$
Q_{1}=\int_{t_{1}}^{t_{2}}\left(\iint_{S} k \operatorname{grad} u \cdot d S\right) d t(2)
$$

The temperature of $V$ changes because of the heat flows in. For a time interval $\left[t_{1}, t_{2}\right]$, if the temperature of every points in $V$ changes from $u\left(x, y, z, t_{1}\right)$ to $u\left(x, y, z, t_{2}\right)$, the heat required in this process is:

$$
\iiint_{V} c \rho\left[u\left(x, y, z, t_{2}\right)-u\left(x, y, z, t_{1}\right)\right] d V
$$

In which $C$ is water's specific heat capacity, $\rho$ is water's density.

Further model. According to heat conservation, the heat input equals to the heat required for the object to raise temperature:

$$
\int_{t_{1}}^{t_{2}}\left[\iint_{S} k \operatorname{grad} u \cdot d S\right] d t=\iiint_{V} c \rho\left[u\left(x, y, z, t_{2}\right)-u\left(x, y, z, t_{1}\right)\right] d V(4)
$$

In this equation, $S$ is a closed curved surface. Assuming function $u$ continuous second-order partial derivative on $x, y, z$ and continuous one-order partial derivative on $n$, transfer u to triple integral according to Gauss Formula [4]:

$\iint_{S} k \operatorname{grad} u \cdot d S=\iiint_{V} k \operatorname{div} \operatorname{grad} u d V=\iiint_{V} k \nabla^{2} u d V$

At the same time, change the volume integral in (4)to:

$$
\iiint_{V} c \rho\left(\int_{t_{1}}^{t_{2}} \frac{\partial u}{\partial t} d t\right) d V=\int_{t_{1}}^{t_{2}}\left[\iiint_{V} c \rho \frac{\partial u}{\partial t} d V\right] d t(6)
$$

Now we have:

$$
\int_{t_{1}}^{t_{2}}\left[\iiint_{V} k \nabla^{2} u d V\right] d t=\int_{t_{1}}^{t_{2}}\left[\iiint_{V} c \rho \frac{\partial u}{\partial t} d V\right] d t
$$

Since time interval $\left[t_{1}, t_{2}\right]$ and space $V$ are arbitrarily sellected, and the integrand is continuous, so (1) works when its integrands are equal: 


$$
\frac{\partial u}{\partial t}=a^{2} \nabla^{2} u=a^{2}\left(\frac{\partial^{2} u}{\partial x^{2}}+\frac{\partial^{2} u}{\partial y^{2}}+\frac{\partial^{2} u}{\partial z^{2}}\right)(8)
$$

In which:

$$
a^{2}=\frac{k}{c \cdot \rho}(9)
$$

\section{Example}

From the analysis above, we get the bathtub water temperature distribution in time and space. Take a rectangular bathtub as an example, we defined the shape of bathtub as $1.7 m \times 0.8 m \times 0.5 m$, the thickness of wall $d$ is $2 \mathrm{~cm}$, the temperature of bathroom $T_{a}$ is $25^{\circ} \mathrm{C}$, the relative humidity in bathroom is $80 \%$, the temperature of adding water $T_{\text {in }}$ is $60^{\circ} \mathrm{C}$, the person in bathtub weight $60 \mathrm{~kg}$ and height $1.7 \mathrm{~m}$.We obtained the bath water temperaturedistribution below.

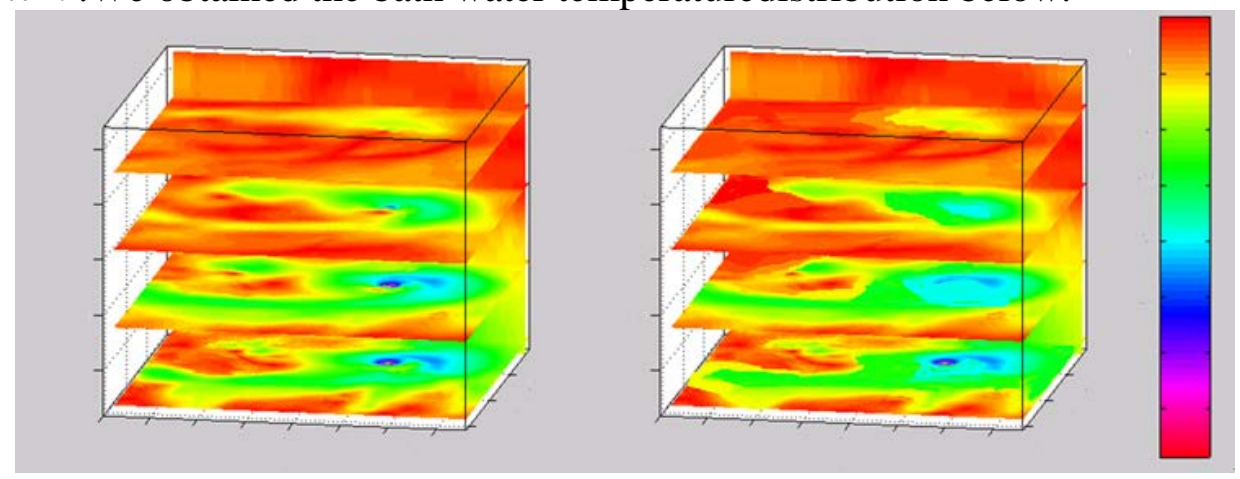

Fig.2 the water temperature distribution

\section{Summary}

The temperature distribution in the bathtub relates to the partial differential equation to three-dimensional, from the mathematical point of direct solution of three-dimensional differential equations have great difficulty. Treatment of practical engineering will often simplify the problem, the three-dimensional processing into three one-dimensional problems, and then solve respectively. By substituting actual data that can be found in the limited space in the bathtub[5], temperature changes with space has certain limitations, often the water near tap will have higher temperature, while in other parts of the water with negligible gap.

According to the conclusions, some methods can be taken to designing best strategy the person in the bathtub can adopt with the combination of the heat loss considered.Through the model, it is also clear about that it is difficult to get an evenly maintained temperature throughout the bath water. Turn on the faucet widely can surely keep the water warm. How to set the amount of water is another research project.

\section{Reference}

[1]Thermal conduction. (2016, January 25). In Wikipedia, The Free Encyclopedia.Retrieved,12:25,February1,2016,from,https://en.wikipedia.org/w/index.php?title=T hermal_conduction\&oldid $=701604560$

[2] Davies T W. FOURIER'S LAW[J]. Atomization \& Sprays, 2006, 79(16):374-379.

[3] Yuanming Wang. Equations of mathematical physics and special functions[M].Beijing: Higher Education Press,2012

[4] Yu, J., Yu, J., \& Yu, J. (1996). The three-parameter gauss formula and its application in mountain precipitation vertical distribution studies. Geographical Research, 15(4), 82-86. 
[5] Varga S, Oliveira J C, Smout C, et al. Temperature distribution analysis of a water cascading retort in rotary and static modes[J]. International Journal of Food Science \& Technology, 2001, 36(5):551-562. 